\title{
Shock tube study of kerosene ignition delay
}

\author{
S. Wang, B.C. Fan, Y.Z. He, and J.P. Cui
}

Key Laboratory of High Temperature Gas Dynamics, Institute of Mechanics, Chinese Academy of Sciences, Beijing 100080, China

\section{Introduction}

The ignition delay time of a fuel is usually concerned as a characteristic time to scale the duration of the gas flow passing through the combustion chamber, which is a criterion to measure the capability of the ignition and sustaining of the flame in the engine [1]. When using the Computational Fluid Dynamics (CFD) to simulate the real process in a combustion chamber, a simplified chemical reaction model is necessary. One of the criteria of the validation of the model is that it should correctly reflect the behavior of the ignition delay time $[2,3]$. The ignition delay time is also a mark to indicate what an additive is more effective to enhance the ignition process for hydrocarbon fuels $[4,5]$.

Kerosene is an important hydrocarbon fuel being used for supersonic combustion. There appears to be very little ignition delay time data available for kerosene. Mullins' data have been used to characterize the ignition behaviors for kerosene [6]. However, Mullins' data have been obtained in a combustion rig fed with hot vitiated air [7]. In addition to chemical characteristics, these data include the effects of many physical factors, such as fuel atomization, and air vitiation. It should be noted that Mullins' data showed fuel atomization has a small effect on the ignition delay for kerosene, but the atomization is known to be important to liquid fuel ignition. From a purely academic viewpoint, ignition delay measurements are necessary, which include only the chemical component of the ignition process and exclude the vitiation effect, because these data are more fundamental and in any case of more widespread application to science generally.

The shock tube is widely used in the study of the ignition delay time of hydrocarbon fuels. Davidson et al. [8] have noted that the uncertainty in the concentration of the fuel vapor due to the adsorption of the fuel vapor on the shock tube wall is one of the error sources in ignition time measurements of liquid fuels. To account for this effect, they put forward a technique to measure the in situ vapor concentration of JP-10 using laser absorption at $3.39 \mu \mathrm{m}$ [9]. However, kerosene is a complex mixture of many hydrocarbon components rather than a single component fuel. To assess the degree of fuel vapor adsorption and enable a more accurate measurement of the fuel vapor concentration in gas phase may be important criteria in determining the reliable ignition delay time. In this study, the gaseous mean concentration of kerosene was determined by directly measuring the vapor pressure of kerosene using a high-precision vacuum gauge, in conjunction with gas chromatography to assess the adsorption contents for the different components of kerosene. Then, the ignition delay times for kerosene diluted in argon were measured under wider conditions. Furthermore, many existing measurements in shock tube have been carried out at the temperature range higher than $1300 \mathrm{~K}$, which is not enough for application in the scramjet engine working at the lower temperature part. The current 
study is concerned with the ignition delay time for kerosene over a wider temperature range, especially extending the lower temperature bound to $1000 \mathrm{~K}$.

\section{Experimental}

The experiments were carried out in a shock tube at the Institute of Mechanics, Chinese Academy of Sciences. The driver section has a length of $2.0 \mathrm{~m}$ and the driven section is $1.8 \mathrm{~m}$ in length. Both have circular cross sections with an inner diameter of $44 \mathrm{~mm}$. The driven section was evacuated down to the ultimate pressure of $1 \times 10^{-2} \mathrm{~Pa}$ by a turbomolecular pump. The outgassing rate was smaller than $5 \times 10^{-3} \mathrm{~Pa} / \mathrm{min}$. The incident shock speeds were measured by two piezoelectric transducers mounted on the shock tube sidewall. The conditions behind the reflected shock were calculated from the incident shock speed using the one-dimensional shock relations, and the contributions of oxidizer and fuel to the specific heat ratio and the sound speed were taken into account. A piezoelectric transducer mounted on the endwall of the driven section was used to monitor the pressure evolution in the reflected shock region. A quartz window was installed on the sidewall very close to the endplate of the driven section to monitor the emission from the ignition process in the reflected shock region. The emission focused through a lens was detected by using a photomultiplier after passing through a monochromator centered at the emission line of $\mathrm{OH}$ radical. The pressure and emission signals were recorded finally by a transient $\mathrm{A} / \mathrm{D}$ transducer. A schematic of the facility is shown in Fig. 1.

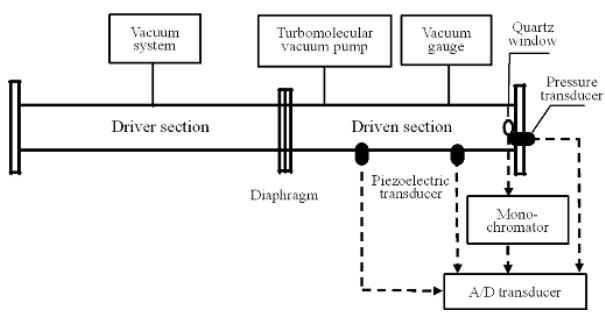

Fig. 1. Schematic of shock tube facility

A longer observation time is required as ignition times increase at the lower temperature region. For the purpose of the present effort the shock tube was run under conditions for a tailored interface, resulting in an observation time of about $6 \mathrm{~ms}$. Therefore, instead of pure $\mathrm{He}$, a mixture of $\mathrm{N}_{2}$ and $\mathrm{He}$ with the specific ratio was used as the driver gas to decrease the sound speed of the region 3 of shock tube and achieve conditions, in which the interaction of the reflected shock with the contact surface produces no secondary reflected wave. Under conditions for a tailored interface, the lower temperature bound of experiments was extended to $1000 \mathrm{~K}$ in the current study.

Kerosene is a complex mixture of many heavy hydrocarbon components. The vapor saturation pressures of heavy hydrocarbons at room temperature are low. There exists a severe adsorption on the wall in shock tube experiments for kerosene, resulting in the uncertainty in determination of the composition of the test gas mixture. The very low vapor pressure of kerosene also limits the experimental concentration range. To minimize the 
degree of adsorption and increase the test vapor pressure, the shock tube and the mixing tank for the mixture of $\mathrm{O}_{2}$ and Ar were preheated and maintained at $343 \mathrm{~K}$ throughout the experiments. The further increase in temperature of preheating shock tube has not been adopted, because of the limitation of the working condition for measurement transducers. Since kerosene is a complex mixture of many heavy hydrocarbon components, the adsorption content of different component differs, leading to the gas composition is different from the liquid composition. A gas chromatograph with a flame ionization detector was used for the measurements of the adsorption content for kerosene. Fig. 2 are the chromatograms, and the left was obtained by directly injecting liquid kerosene sample into the gas chromatograph. After injecting a liquid kerosene sample into the shock tube and mixing fully with Ar, then a gas sample from the shock tube was introduced into the gas chromatograph, the right chromatogram in Fig. 2 was obtained, showing the decrease for peaks of retention times longer than $22 \mathrm{~min}$. Fig. 2 indicates clearly the difference in the adsorption content of different component for kerosene on the shock tube wall. The components of longer retention times have the higher boiling points and adsorb more easily on the shock tube wall.

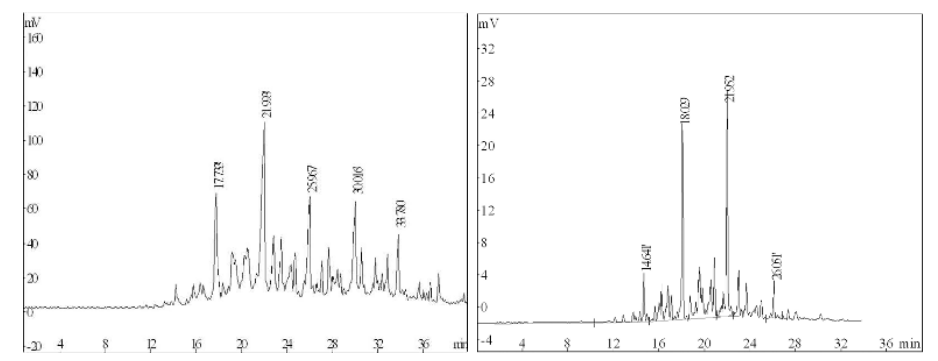

Fig. 2. Chromatograms for kerosene obtained both by directly injecting a liquid sample into the gas chromatograph(left) and when a gas sample from the shock tube was introduced into the gas chromatograph(right)

In the present study, a simulant modified fuel for kerosene was prepared by adding some heavy hydrocarbon components into the original kerosene in proportion to the adsorption content to compensate the loss in the gas phase through the adsorption on the wall. Thus the gas composition of the modified fuel was almost same as the original kerosene. In the current study, the ignition delay measurements were made for this modified kerosene, and the mean gas concentration for kerosene was determined by directly measuring the kerosene vapor pressure and using one-formula surrogate $\mathrm{C}_{10} \mathrm{H}_{22}$ to represent kerosene. After the shock tube was filled with kerosene, the kerosene vapor pressures in adsorption equilibrium were measured directly by using a high-precision vacuum gauge. A membrane vacuum gauge (Beijing Vacuum Instrument Factory, Model ZDM-1) was selected to measure the kerosene pressure, with a resolution of $0.1 \mathrm{~Pa}$ when the working pressure is under $1000 \mathrm{~Pa}$. The time interval from injecting kerosene to the diaphragm rupture was about 10min. To estimate the adsorption extent, the adsorption curves of kerosene in the driven section maintained at $343 \mathrm{~K}$ were measured during $10 \mathrm{~min}$ after injecting $10 \mu \mathrm{L}, 20 \mu \mathrm{L}$ and $30 \mu \mathrm{L}$ of liquid kerosene, respectively, the results are shown in Fig. 3. These curves show the adsorption equilibriums are achieved almost in $5 \mathrm{~min}$ and completely in 10min. 


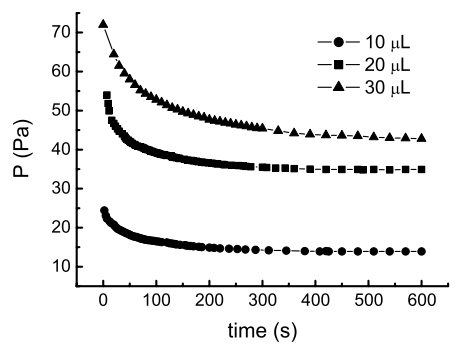

Fig. 3. Adsorption curves of the modified kerosene

After the driven section was evacuated to the ultimate pressure, a quantitative liquid kerosene was injected into the driven section, then the pressure was measured 5 min after the fuel evaporated and adsorbed. Then, the mixture of $\mathrm{O}_{2}$ (99.995\% pure) and $\mathrm{Ar}$ (99.99\% pure) as diluent was added into the driven section, and an additional time of 5 min was allowed for the gases to mix fully, and the final total pressure was measured $10 \mathrm{~min}$ after injecting kerosene. Our experience shows that $5 \mathrm{~min}$ is sufficient to mix the gases fully. Thus the kerosene concentrations could be determined by using the direct pressure measurements based on the adsorption curves. The time interval of $10 \mathrm{~min}$ from injecting fuel to the diaphragm rupture was specified to ensure both achievement of kerosene adsorption equilibrium and homogeneity of the test mixture. The driver section was simultaneously filled with $\mathrm{N}_{2}$ and $\mathrm{He}$ at a required ratio as the tailored interface operation is achieved.

Experiments were performed over the pressure range of 1.8-5.0atm, the temperature range of $1030-1860 \mathrm{~K}$, and fuel concentrations of $0.1-0.33 \%$ mole fraction. The total pressure and the emission of $\mathrm{OH}$ radical at $309 \mathrm{~nm}$ in the reflected shock region were recorded after the bursting of the diaphragm. The radical emission was used as the marker to identify the ignition time. An example of pressure and $\mathrm{OH}$ emission data is shown in Fig. 4. $\mathrm{P}_{5}$ indicates the passing of the reflected shock and the pressure evolution, and

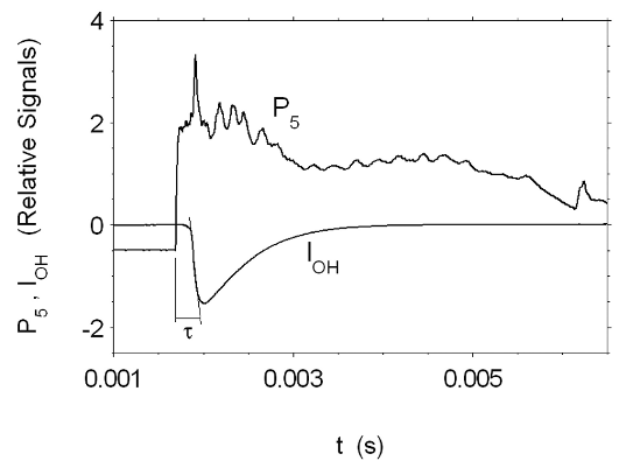

Fig. 4. Example of $\mathrm{OH}$ emission and pressure data. Reflected shock conditions: $1458 \mathrm{~K}, 0.12 \%$ kerosene, $6.5 \% \mathrm{O}_{2}$, and $93.4 \% \mathrm{Ar}$ 
$\mathrm{I}_{O H}$ indicates the $\mathrm{OH}$ emission evolution in the reflected shock region. It can be seen that the abrupt increases in pressure and emission occur at the same time, and the onset of ignition is unequivocal, especially compared to the more conventional determination from the abrupt increase in pressure [10]. The ignition delay time is defined as the time interval between the passing of the reflected shock and the onset of ignition, which is usually determined by fitting a straight line to the initial rapid rising portion of the emission and extrapolating until it intersects with zero, as shown in Fig. 4.

\section{Results}

Ignition delay times were measured for four different fuel $/ \mathrm{O}_{2} / \mathrm{Ar}$ mixtures with stoichiometric ratios of $\phi=2.0,1.0,0.5$ and 0.25 , respectively. The initial compositions of the diluent gases and the injection volumes of kerosene are given in Table 1.

Table 1. Initial composition

\begin{tabular}{llll}
\hline No. & Composition of diluent gas & Injection volume of kerosene & $\phi$ \\
\hline 1 & $2.5 \% \mathrm{O}_{2}+97.5 \% \mathrm{Ar}$ & $30 \mu \mathrm{L}$ & 2.0 \\
2 & $5.0 \% \mathrm{O}_{2}+95.0 \% \mathrm{Ar}$ & $30 \mu \mathrm{L}$ & 1.0 \\
3 & $8.0 \% \mathrm{O}_{2}+92.0 \% \mathrm{Ar}$ & $20 \mu \mathrm{L}$ & 0.5 \\
4 & $6.5 \% \mathrm{O}_{2}+93.5 \% \mathrm{Ar}$ & $10 \mu \mathrm{L}$ & 0.25 \\
\hline
\end{tabular}

The kerosene ignition delay time data are shown in a logarithmic plot of the ignition time vs the reciprocal temperature in Fig. 5. The slopes of the ignition curves are almost the same with increasing $\phi$, suggesting that the ignition activation energy varies only slightly. A least square analysis was performed to correlate the kerosene ignition time data with temperature and concentrations of kerosene and oxygen. For the temperature range of $1030-1870 \mathrm{~K}$, the correlation was obtained with an $R^{2}$ correlation coefficient of 0.990 in the following form

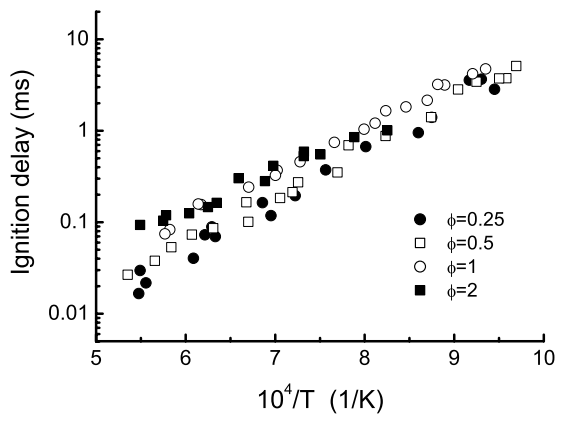

Fig. 5. Ignition delay times for kerosene 


$$
\tau=2.58 \times 10^{-7}[\text { Kerosene }]^{0.42}\left[O_{2}\right]^{-0.40} \exp \left(\frac{100663}{R T}\right)
$$

where $\tau$ is in seconds, $\phi$ is the stoichiometric ratio, [Kerosene $]$ and $\left[\mathrm{O}_{2}\right]$ are in $\mathrm{mol} / \mathrm{cm}^{3}$, and the activation energy is in $\mathrm{J} / \mathrm{mol}$.

The ignition delay time correlation for kerosene shows a relatively weak power dependency (-0.4) for oxygen, but the kerosene concentration power dependency (0.42) is the same as other single-component heavy hydrocarbon fuels that have a strong oxygen power dependency of about $-1.0[11]$.

Acknowledgement. This work was supported by the National Natural Science Foundation of China (Grant No. 90305021).

\section{References}

1. Tishkoff J. M., Drummond J. P., Edwards T., Nejad A. S., Future direction of supersonic combustion research: Air Force/NASA workshop on supersonic combustion, AIAA Paper 97-1017(1997)

2. Held T. J., Marchese A. J., Dryer F. L., A semi-empirical reaction mechanism for n-heptane oxidation and pyrolysis, Combust. Sci. and Technol., 123, 107(1997)

3. Li S. C., Varatharajan B., Williams F. A., Chemistry of JP-10 ignition, AIAA J., 39(12), 2351(2001)

4. Davidson D. F., Horning D. C., Hanson R. K. Hitch B., Shock tube ignition time measurements for n-Heptane/ $\mathrm{O}_{2} / \mathrm{Ar}$ mixtures with and without additives, Proceedings of the 22nd International Symposium on Shock Waves, Imperial College, London, UK, 191(1999)

5. Sidhu S. S., Graham J. L., Kirk D. C., Maurice L. Q., Investigation of effect of additives on ignition characteristics of jet fuels:JP-7 and JP-8, Proceedings of the 22nd International Symposium on Shock Waves, Imperial College, London, UK, 285(1999)

6. Veretennicov V. G., Autoignition study on kerosene in supersonic flow, Moscow Aviation Institute, SPC 96-4089, Moscow, Russia, 1997

7. Mullins B. P., Studies on the spontaneous ignition of fuels injected into a hot air-stream part 2: Effect of physical factors upon the ignition delay of kerosene-air mixtures, Fuel, 32, 234(1953)

8. Davidson D. F., Horning D. C., Hanson R. K., Shock tube ignition time measurements for n-Heptane $/ \mathrm{O}_{2} / \mathrm{Ar}$ and JP-10/O $/ \mathrm{O}_{2} /$ Ar mixtures, AIAA Paper 99-2216(1999)

9. Davidsion D. F., Horning D. C., Herbon J. T., Hanson R. K., Shock tube measurements of JP-10 ignition, Proc. Combust. Inst., 28, 1687(2000)

10. Brown C. J., Thomas G. O., Experimental studies of shock-induced ignition and transition to detonation in ethylene and propane mixtures, Combust. Flame,117, 861(1999)

11. Colket M. B., Spadaccini L. J., Scramjet fuels autoignition study, J. Prop. Power, 17(2), $315(2001)$ 